MATHEMATICS OF COMPUTATION

Volume 69, Number 229, Pages 325-337

S 0025-5718(99)01204-1

Article electronically published on August 23, 1999

\title{
THE POSTAGE STAMP PROBLEM: AN ALGORITHM TO DETERMINE THE $h$-RANGE ON THE $h$-RANGE FORMULA ON THE EXTREMAL BASIS PROBLEM FOR $k=4$
}

\author{
SVEIN MOSSIGE
}

Abstract. Given an integral "stamp" basis $A_{k}$ with $1=a_{1}<a_{2}<\ldots<$ $a_{k}$ and a positive integer $h$, we define the $h$-range $n\left(h, A_{k}\right)$ as

$$
n\left(h, A_{k}\right)=\max \left\{N \in \mathbf{N} \mid n \leq N \Longrightarrow n=\sum_{1}^{k} x_{i} a_{i}, \sum_{1}^{k} x_{i} \leq h, n, x_{i} \in \mathbf{N}_{0}\right\}
$$

$\mathbf{N}_{0}=\mathbf{N} \cup\{0\}$. For given $h$ and $k$, the extremal basis $A_{k}^{*}$ has the largest possible extremal $h$-range

$$
n(h, k)=n\left(h, A_{k}^{*}\right)=\max _{A_{k}} n\left(h, A_{k}\right) .
$$

We give an algorithm to determine the $h$-range. We prove some properties of the $h$-range formula, and we conjecture its form for the extremal $h$-range. We consider parameter bases $A_{k}=A_{k}(h)$, where the basis elements $a_{i}$ are given functions of $h$. For $k=4$ we conjecture the extremal parameter bases for $h \geq 11385$.

\section{BACKGRound}

Given an integral basis $A_{k}=\left\{a_{1}, a_{2}, \ldots, a_{k}\right\}$ with $a_{1}=1<a_{2}<\ldots<a_{k}$ and a positive integer $h$, we define the $h$-range $n\left(h, A_{k}\right)$ as

$$
n\left(h, A_{k}\right)=\max \left\{N \in \mathbf{N} \mid n \leq N \Longrightarrow n=\sum_{1}^{k} x_{i} a_{i}, \sum_{1}^{k} x_{i} \leq h, n, x_{i} \in \mathbf{N}_{0}\right\} .
$$

$\mathbf{N}_{0}=\mathbf{N} \cup\{0\}$. The integer $n \in \mathbf{N}$ has an $h$-representation by $A_{k}$ if

$$
n=\sum_{1}^{k} x_{i} a_{i} \mid \sum_{1}^{k} x_{i} \leq h, x_{i} \in \mathbf{N}_{0} .
$$

We consider only bases $A_{k}$ which are $h$-admissible, that is,

$$
a_{k} \leq n\left(h, A_{k}\right) .
$$

For given $h$ and $k$, the extremal basis $A_{k}^{*}$ has the largest possible extremal $h$-range

$$
n(h, k)=n\left(h, A_{k}^{*}\right)=\max _{A_{k}} n\left(h, A_{k}\right) .
$$

A popular interpretation arises if we consider the integers $a_{i}$ as stamp denominations and $h$ as the "size of the envelope." More information about the "postage

Received by the editor March 13, 1996.

1991 Mathematics Subject Classification. Primary 11B13, $11 \mathrm{D} 85$.

(C)1999 American Mathematical Society 
stamp problem" can be found in E. S. Selmer's comprehensive research monograph [17]. Here we mainly use Selmer's notation and presentation.

In the beginning, the main interest was centered around the global aspect, to find an extremal basis $A_{k}^{*}$ with extremal $h$-range. The "local" aspect is: Determine $n\left(h, A_{k}\right)$ when $h, k$ and a particular basis $A_{k}$ are given.

In the global case, a convenient approach is to keep $k$ fixed and let $h$ increase, asking for asymptotic values of the extremal $h$-range $n(h, k)$. We can also ask for asymptotic values of "local" $h$-ranges $n\left(h, A_{k}\right)=n\left(h, A_{k}(h)\right)$, when the basis elements $a_{i}$ are given functions of $h$. We shall call such bases $A_{k}(h)$ parameter bases.

Let $\varphi$ be the prefactor defined by

$$
n\left(h, A_{k}(h)\right)=\varphi\left(\frac{h}{k}\right)^{k}(1+\mathrm{o}(1)) .
$$

Both the local and the global problems are trivial for $k=2$, Stöhr [20]. The extremal bases $A_{3}^{*}$ were determined by Hofmeister [4], [5]. For $k \geq 4$, our knowledge is much more limited. The best known general upper bound is due to Rødseth [15]:

$$
n(h, k) \leq \frac{(k-1)^{k-2}}{(k-2) !}\left(\frac{h}{k}\right)^{k}+\mathcal{O}\left(h^{k-1}\right) .
$$

For $k=4$, the prefactor $\varphi=4.5$ is far too large, and Kirfel [7] has the strongest published result:

$$
n(h, 4) \leq 2.35\left(\frac{h}{4}\right)^{4}+\mathcal{O}\left(h^{3}\right)
$$

In [12] the author proved the lower bound

$$
n(h, 4) \geq 2.008\left(\frac{h}{4}\right)^{4}+\mathcal{O}\left(h^{3}\right) .
$$

The proof consists in determining a parameter basis $A_{4}=A_{4}(h)$ whose $h$-range equals the bound given. However (May 1991, unpublished), Kirfel and the author have shown that the lower bound $2.008 \ldots$ (more decimals in (32)) is really sharp. Hence, it is natural to investigate the local extremal parameter bases for $k=4$.

For $k=5$, Kolsdorf in [6] has given a parameter basis with asymptotic $h$-range $3.06(h / 5)^{5}$.

It was shown by Kirfel [8] that the limit

$$
c_{k}=\lim _{h \rightarrow \infty} n(h, k) /(h / k)^{k}
$$

really exists for all $k \geq 2$. It is known that $c_{2}=1, c_{3}=4 / 3$, and $c_{4}=2.008 \ldots$

Looking for the extremal bases, we consider parameter bases $A_{k}(h)$ for which

$$
n\left(h, A_{k}(h)\right) \text { has order of magnitude } h^{k} .
$$

For the basis elements, this implies that $a_{i}(h)$ has order of magnitude $h^{i-1}, i=$ $2,3, \ldots, k$.

Representations and gain. The regular representation of $n$ by $A_{k}$,

$$
n=\sum_{1}^{k} e_{i} a_{i},
$$


satisfies the conditions

$$
e_{1}+e_{2} a_{2}+\ldots+e_{j} a_{j}<a_{j+1}, \quad j=1,2, \ldots, k-1 .
$$

A representation of $n$ is minimal if the number of addends is the smallest possible among all representations. For the elements $a_{i} \in A_{k}, i=2,3, \ldots, k$, we write

$$
a_{i}=\gamma_{i-1} a_{i-1}-\sum_{j=1}^{i-2} \beta_{j}^{(i)} a_{j},
$$

where $\gamma_{i-1}=\left\lceil a_{i} / a_{i-1}\right\rceil \geq 2$, and $\sum_{j=1}^{i-2} \beta_{j}^{(i)} a_{j}=\gamma_{i-1} a_{i-1}-a_{i}$ is the regular representation by $A_{i-2}$. As usual, $\lceil x\rceil$ denotes the smallest integer $\geq x \in \mathbf{R}$. Hofmeister [5] calls (6) the normal form of the basis $A_{k}$. Let $n \in \mathbf{N}$ have a regular representation (4) by $A_{k}$, and let $s_{i} \in \mathbf{Z}, i=2,3, \ldots, k$. From (6) we get a new representation $n=\sum z_{j} a_{j}$ by an $\left(s_{2}, s_{3}, \ldots, s_{k}\right)$-transfer:

$$
\begin{aligned}
n & =\sum_{i=1}^{k} e_{i} a_{i}+\sum_{i=2}^{k} s_{i}\left(\gamma_{i-1} a_{i-1}-a_{i}-\sum_{j=1}^{i-2} \beta_{j}^{(i)} a_{j}\right) \\
& =\sum_{j=1}^{k}\left(e_{j}-s_{j}+s_{j+1} \gamma_{j}-\sum_{i=j+2}^{k} s_{i} \beta_{j}^{(i)}\right) a_{j}=\sum_{j=1}^{k} z_{j} a_{j},
\end{aligned}
$$

with $s_{1}=s_{k+1}=\gamma_{k}=0$. We say that the transfer is possible if $z_{j} \geq 0, \quad j=$ $1, \ldots, k$.

The sum of the reductions in the coefficients is the gain $G\left(s_{2}, s_{3}, \ldots, s_{k}\right)$ in the transfer:

$$
G\left(s_{2}, s_{3}, \ldots, s_{k}\right)=\sum_{j=1}^{k}\left(e_{j}-z_{j}\right) .
$$

The usefulness of such transfers stems from the following result of Hofmeister [5]: Every "legal" representation $n=\sum z_{i} a_{i}\left(z_{i} \geq 0\right)$ can be obtained from the regular representation by a suitable $\left(s_{2}, s_{3}, \ldots, s_{k}\right)$-transfer with all $s_{i} \geq 0$. We also cite another result of Hofmeister [5]: If a parameter basis $A_{k}(h)$ satisfies (3) and is expressed in normal form (6), then the $s_{i}$ of any possible $\left(s_{2}, s_{3}, \ldots, s_{k}\right)$-transfer are bounded as $h \rightarrow \infty$. See also Kirfel [7].

In 1963, Hofmeister [5], [3] gave formulas for the regular $h$-range of a basis. If only regular $h$-representations are allowed, we get the regular $h$-range. He also conjectured the formula for the extremal regular $h$-range, later proved by Mrose [14].

Let

$$
h_{0}=h_{0}\left(A_{k}\right)=\min \left\{h \in \mathbf{N} \mid a_{k} \leq n\left(h, A_{k}\right)\right\} .
$$

For all $k$ and $h \geq h_{0}$ we trivially have

$$
n\left(h+1, A_{k}\right) \geq n\left(h, A_{k}\right)+a_{k} .
$$

Furthermore, Selmer [17] proved that, for arbitrary $k$ and $h \geq h_{0}$,

$$
n\left(h, A_{k}\right) \geq(h+1) a_{k-1}-a_{k}
$$

implies

$$
n\left(h+1, A_{k}\right)=n\left(h, A_{k}\right)+a_{k} .
$$


If $h$ is increased by 1 , the right-hand side of (10) increases with $a_{k-1}$, while the left-hand side increases with at least $a_{k}$. There is consequently an $h_{1}\left(\geq h_{0}\right)$ such that (10) and hence (11) are satisfied for all $h \geq h_{1}$. This means that for given $h, h \geq h_{1}$, we have

$$
n\left(h, A_{k}\right)=n\left(h_{1}, A_{k}\right)+\left(h-h_{1}\right) a_{k} .
$$

We see that for a basis $A_{k}$ there may be different $h$-range formulas according to the value of $h, h_{0} \leq h \leq h_{1}$. From (12), the $h$-range formula is the same for all $h \geq h_{1}$. In looking for bases with large $h$-range, we often have the same $h$-range formula for all $h \geq h_{0}$.

Lemma 1. Let the basis $A_{k}$ and the possible transfers $T^{(i)}=\left(s_{2}^{(i)}, s_{3}^{(i)}, \ldots, s_{k}^{(i)}\right)$, $i=1,2, \ldots, \eta$, be given. Let

$$
h_{2}=\min \left\{h \mid n\left(h, A_{k}\right) \geq \max _{i}\left\{s_{k}^{(i)}\right\} a_{k}\right\} .
$$

Then for $h \geq h_{2}$

$$
n\left(h, A_{k}\right)=n\left(h_{2}, A_{k}\right)+\left(h-h_{2}\right) a_{k} .
$$

Proof. The minimal representation of a positive integer is independent of the value of $h$. For $h \geq h_{2}$ we can use all the transfers. From above we know that for $h \geq h_{1}$ the $h$-range is determined by (12) and we have $h_{1} \leq h_{2}$. Note that only the transfers actually used determine $h_{2}$.

\section{ThE $h$-RANGE ALGORITHM}

In the literature we find more or less general $h$-range algorithms by Lunnon [9], Riddell and Chan [16], Mossige [10], and Challis [2].

Let the basis $A_{4}$ and the possible transfers be given. For each integer $n \in$ $\left[1, n\left(h, A_{4}\right)\right]$ given in a regular representation $\sum e_{j} a_{j}$, we use the possible transfer with the largest gain to give the minimal representation of $n, \sum z_{j} a_{j}$. It satisfies the inequality $\sum e_{j}-$ gain $=\sum z_{j} \leq h$. The algorithm gives sufficient such inequalities that express the conditions that all the integers $n$ have an $h$-representation. The least integer $n$ with $n+1$ not having an $h$-representation is the $h$-range. For a given basis, the algorithm determines $h_{0}$ and from which $h \geq h_{0}$ the $h$-range formula is the same. The result is valid for all $h \geq h_{0}$.

We give the algorithm for $k=4$, but it may be generalized to $k>4$.

Now, let the possible transfers $T^{(i)}=\left(s_{2}^{(i)}, s_{3}^{(i)}, s_{4}^{(i)}\right), i=1, \ldots, \eta$, for the basis $A_{4}$ be given. Then the minimal representation of an integer $n>0$ is independent of $h$.

The upper bounds for the $e_{j}$ 's are given such that the representation (4) is regular. The conditions for the transfers to be possible give lower bounds for the $e_{j}$ 's. The coefficients $z_{j}$ of $(7)$ must be $\geq 0$, giving lower bounds on the $e_{j}$ 's. The gain (reduction of coefficient sum) must be positive.

We get the following values of the gain and the lower bounds for $e_{j}$ 's:

$$
\begin{aligned}
G_{i}= & s_{2}^{(i)}\left(-\gamma_{1}+1\right)+s_{3}^{(i)}\left(\beta_{1}^{(3)}-\gamma_{2}+1\right) \\
& +s_{4}^{(i)}\left(\beta_{1}^{(4)}+\beta_{2}^{(4)}-\gamma_{3}+1\right) \geq 1,
\end{aligned}
$$




$$
\begin{aligned}
& e_{1} \geq-s_{2}^{(i)} \gamma_{1}+s_{3}^{(i)} \beta_{1}^{(3)}+s_{4}^{(i)} \beta_{1}^{(4)}=L_{i}^{\prime}, \\
& e_{2} \geq s_{2}^{(i)}-s_{3}^{(i)} \gamma_{2}+s_{4}^{(i)} \beta_{2}^{(4)}=M_{i}^{\prime}, \\
& e_{3} \geq s_{3}^{(i)}-s_{4}^{(i)} \gamma_{3}=N_{i}^{\prime}, \\
& e_{4} \geq s_{4}^{(i)}=Q_{i} .
\end{aligned}
$$

We may, however, find $L_{i}^{\prime}$ and/or $M_{i}^{\prime}$ and/or $N_{i}^{\prime}<0$, and operate instead with lower bounds

$$
e_{1} \geq L_{i}=\max \left\{0, L_{i}^{\prime}\right\}, e_{2} \geq M_{i}=\max \left\{0, M_{i}^{\prime}\right\}, e_{3} \geq N_{i}=\max \left\{0, N_{i}^{\prime}\right\} .
$$

There may be repetitions among the $L_{i}, M_{i}, N_{i}$, or $Q_{i}$. We sort them first without repetitions:

$$
\begin{aligned}
& 0=L_{0}<L_{1}<\ldots<L_{r_{1}}<L_{r_{1}+1}=U_{1}+1 \\
& 0=M_{0}<M_{1}<\ldots<M_{r_{2}}<M_{r_{2}+1}=U_{2}+1 \\
& 0=N_{0}<N_{1}<\ldots<N_{r_{3}}<N_{r_{3}+1}=U_{3}+1 \\
& 0=Q_{0}<Q_{1}<\ldots<Q_{r_{4}}<Q_{r_{4}+1}=U_{4}+1 .
\end{aligned}
$$

The numbers $L_{0}, L_{r_{1}+1}$, and so forth, are added. Here $L_{0}=M_{0}=N_{0}=Q_{0}=$ $G_{0}=0$ corresponds to using the regular representation itself, hence no transfer. The upper bounds for $e_{j}$, say $U_{j}, j=1,2,3$, are given such that the representation (4) is regular. For $e_{4}$ we note that the largest $s_{4}^{(i)}$ is $<h$, and we put $U_{4}=h$. Then we sort all the gains $G_{i}$,

$$
G^{(1)} \geq G^{(2)} \geq \ldots \geq G^{(\eta)}>0,
$$

without registering possible equalities. This gives a sequence of quintuples

$$
\left(G^{(i)}, L^{(i)}, M^{(i)}, N^{(i)}, Q^{(i)}\right), \quad i=1,2, \ldots, \eta,
$$

to which we add $(0,0,0,0,0)$, corresponding to no transfer. Assume that $p, q, r$ and $s$ are given such that

$$
0 \leq p \leq r_{1}, \quad 0 \leq q \leq r_{2}, \quad 0 \leq r \leq r_{3}, \quad 0 \leq s \leq r_{4} .
$$

Let $e_{1}, e_{2}, e_{3}$ and $e_{4}$ be given such that

$$
\begin{aligned}
& L_{p} \leq e_{1} \leq L_{p+1}-1, M_{q} \leq e_{2} \leq M_{q+1}-1, \\
& N_{r} \leq e_{3} \leq N_{r+1}-1, Q_{s} \leq e_{4} \leq Q_{s+1}-1 .
\end{aligned}
$$

We then scan the quintuples $\left(G^{(i)}, L^{(i)}, M^{(i)}, N^{(i)}, Q^{(i)}\right), i=1,2, \ldots, \eta+1$, and register the first time (largest gain) such that

$$
L^{(i)}<L_{p+1}, \quad M^{(i)}<M_{q+1}, \quad N^{(i)}<N_{r+1}, \quad Q^{(i)}<Q_{s+1} .
$$

The corresponding gain $G^{(i)}=G_{p q r s}$ is then the largest one which can be used in the case (16), (17). We must always have

$$
e_{1}+e_{2}+e_{3}+e_{4}-G^{(i)} \leq h .
$$

In the "worst" case $e_{1}=L_{p+1}-1, e_{2}=M_{q+1}-1, e_{3}=N_{r+1}-1$, and the corresponding integer $n$ has the regular representation

$$
n=L_{p+1}-1+\left(M_{q+1}-1\right) a_{2}+\left(N_{r+1}-1\right) a_{3}+e_{4}^{\prime} a_{4},
$$

with

$$
L_{p+1}-1+M_{q+1}-1+N_{r+1}-1+e_{4}^{\prime}-G_{p q r s} \leq h .
$$


If $Q_{s+1}<U_{4}+1$, then $e_{4}^{\prime}=Q_{s+1}-1$, and we must have

$$
L_{p+1}-1+M_{q+1}-1+N_{r+1}-1+Q_{s+1}-1-G_{p q r s} \leq h .
$$

The inequality defines a lower bound for $h$. If $Q_{s+1}=U_{4}+1$, then

$$
e_{4}^{\prime}=h-\left(L_{p+1}-1+M_{q+1}-1+N_{r+1}-1-G_{p q r s}\right)
$$

gives an upper bound for $e_{4}^{\prime}$.

Each subset with $Q_{s+1}=U_{4}+1$ determines a value $e_{4}^{\prime}$ such that all values $n=\sum e_{j} a_{j}$ satisfying (16), (17) have $h$-representations, and the value

$$
n^{\prime}=L_{p+1}-1+\left(M_{q+1}-1\right) a_{2}+\left(N_{r+1}-1\right) a_{3}+\left(e_{4}^{\prime}+1\right) a_{4}
$$

does not, but all other values

$$
m^{\prime}=e_{1}+e_{2} a_{2}+e_{3} a_{3}+\left(e_{4}^{\prime}+1\right) a_{4}
$$

where $e_{1}, e_{2}, e_{3}$ satisfy (16), (17), do. Let

$$
m=\min _{p q r}\left\{n^{\prime}\right\}
$$

then $m$ has no $h$-representation, but all values less than $m$ do, so the $h$-range $n\left(h, A_{k}\right)=m-1$.

If $Q_{s+1}<U_{4}+1$, then $e_{4}^{\prime}=Q_{s+1}-1 \geq 0$, and the inequality (20) defines a lower bound for $h$. Then $h_{0}$ is the minimal value of $h$ that satisfies the inequalities (20) in all the cases with $e_{4}^{\prime}=Q_{s+1}-1=0$. Let $h_{3}$ be the minimal value of $h$ such that all the inequalities (20) are satisfied. Then for $h \geq h_{3}$ the $h$-range $n\left(h, A_{4}\right) \geq \max _{i}\left\{s_{4}^{(i)}\right\} a_{4}$, with the index running over all used transfers. From Lemma 1 , the basis has the same $h$-range formula for all $h \geq h_{3}$. If $h_{3}>h_{0}$ and $h_{0} \leq h \leq h_{3}$, then the $h$-range is $m-1,(23)$.

For given $h \geq h_{3}$, the upper bound on $e_{4}^{\prime}$ is

$$
h-\max \left(L_{p+1}-1+M_{q+1}-1+N_{r+1}-1-G_{p q r s}\right),
$$

where the maximum is taken over all the cases with $Q_{s+1}=U_{4}+1$, see $(21)$. One may also use $e_{4}^{\prime}$ to determine the prefactor of the basis; see [12] and Selmer [19].

Let $h \geq h_{3}$. The integers $n \in\left[0, h a_{4}\right]$ given in regular representation with an $h$-representation may be split into disjoint sets. For each set of integers we perform the procedure above. Let $N$ be the smallest one of the integers $m-1,(23)$ with $Q_{s+1}=U_{4}+1$. Since we have used the possible transfer with the largest gain for each integer, $N$ is the $h$-range of the basis.

The algorithm may be easily modified for a parameter basis $A_{4}(h)$ where $\gamma$, $\beta, G, L, M, N$ and $U$ are either linear expressions in $h$ of the form $c h+d$ or constants. This means that the comparisons may have to be done in two steps. Let $L_{1}=c_{1} h+d_{1}$ and $L_{2}=c_{2} h+d_{2}$. Then if $c_{1} \neq c_{2}$ we are finished with one comparison. If $c_{1}=c_{2}$ we have to compare $d_{1}$ with $d_{2}$ also.

We have described a constructive procedure to determine the $h$-range of a given explicit basis $A_{4}$ or a parameter basis $A_{4}(h)$ with a given set of transfers.

In [12] the author used the algorithm for $k=4$ to determine the $h$-range formulas of the parameter basis that by optimization gave the asymptotic prefactor $c_{4}$. Also it contributes to the characterization of the $h$-range formulas.

The algorithm requires that all the subsets (16), (17) must be considered in turn. A slightly different approach might reduce the number of subsets which need to be considered. 
First, choose $Q_{s} \leq e_{4} \leq Q_{s+1}-1$. Now extract from the set of quintuples $\left(G^{(i)}, L^{(i)}, M^{(i)}, N^{(i)}, Q^{(i)}\right)$ just those which satisfy $Q^{(i)}<Q_{s+1}$. We do not need to consider other transfers, because they are not possible for these values of $e_{4}$. This set of quintuples defines new subdivisions for $e_{3}$, and there will in general be fewer subdivisions than before. Next, we choose one of these subdivisions $N_{r} \leq e_{3} \leq N_{r+1}-1$, and repeat the process. Finally, when we have chosen subdivisions for $e_{4}, e_{3}, e_{2}$ and $e_{1}$ we will have a set of quintuples that describes precisely those transfers which are possible for the subset, and so we have only to choose the one with highest gain.

Properties of the $h$-range formula. Since Hofmeister [5] gave explicit formulas for the regular $h$-range of a basis, we assume that at least one transfer must be applied.

Theorem 1. Let $h, k \geq 3$, and let the admissible basis $A_{k}$ be given in normal form (6). Let $\sum_{1}^{k} \epsilon_{i} a_{i}=n\left(h, A_{k}\right), \epsilon_{i} \in \mathbf{N} \cup\{0\}$, be the regular representation of the $h$-range. Let us assume $\epsilon_{1}<a_{2}-2$. Then

$$
\epsilon_{1}=\sum_{i=3}^{k} s_{i} \beta_{1}^{(i)}-s_{2} \gamma_{1}-2,
$$

where $\left(s_{2}, \ldots, s_{k}\right)$ is one of the transfers used for $A_{k}$. For this transfer to be possible for an integer with regular representation $\sum e_{j} a_{j}$, it is at least necessary that

$$
e_{1} \geq \sum_{i=3}^{k} s_{i} \beta_{1}^{(i)}-s_{2} \gamma_{1}
$$

Proof. Let $n\left(h, A_{k}\right)=N$. The integer $N+1$ has no $h$-representation. Consider the integer $N+2=\sum \epsilon_{i} a_{i}+2$. Since the basis is admissible, we have one coefficient $\epsilon_{j} \geq 1, j \in[2, k]$. Then the integer

$$
M=N+2-a_{j}=\epsilon_{1}+2+\sum_{2}^{j-1} \epsilon_{i} a_{i}+\left(\epsilon_{j}-1\right) a_{j}+\sum_{j+1}^{k} \epsilon_{i} a_{i}=\sum_{1}^{k} z_{i}^{\prime} a_{i},
$$

and the representation is regular with $\sum_{1}^{k} z_{i}^{\prime}>h . M$ has an $h$-representation

$$
M=\sum_{1}^{k} z_{i} a_{i} \text { with } \sum_{1}^{k} z_{i} \leq h, \text { since } M \leq N .
$$

If $\left(s_{2}, \ldots, s_{k}\right)$ is the transfer between the two representations for $M$, we have at least one $s_{j}>0, j \in[2, k]$. The $h$-representation of $M$ can not be used for $M-1$, since $N+1=M-1+a_{j}$ would then have an $h$-representation. Hence the representation of $M$ must have $z_{1}=0$, and thus from (7) (with $e_{j}$ replaced by $z_{j}^{\prime}$ )

$$
0=z_{1}^{\prime}-0+s_{2} \gamma_{1}-\sum_{3}^{k} s_{i} \beta_{1}^{(i)}=\epsilon_{1}+2+s_{2} \gamma_{1}-\sum_{3}^{k} s_{i} \beta_{1}^{(i)} .
$$

From the $h$-range algorithm and Theorem 1 we have

Theorem 2. Let $k=4, h \geq 3$, and let the admissible basis $A_{4}$ be given in normal form (6) with $\gamma_{2} \geq 3, \beta_{2}^{(4)} \geq 1$ and $2 a_{2}>\beta_{1}^{(3)}+\beta_{1}^{(4)}>a_{2}$. Let the used transfers 
of the basis be $T^{(j)}=\left(s_{2}^{(j)}, s_{3}^{(j)}, s_{4}^{(j)}\right), j=1, \ldots, \eta$. Let the regular representation of the h-range of the basis be

$$
n=\epsilon_{1}+\epsilon_{2} a_{2}+\ldots+\epsilon_{4} a_{4} .
$$

Then

$$
\begin{gathered}
\epsilon_{1}=\left\{\begin{array}{l}
\sum_{i=3}^{4} s_{i}^{\left(j_{1}\right)} \beta_{1}^{(i)}-s_{2}^{\left(j_{1}\right)} \gamma_{1}-2, \\
\gamma_{1}-2,
\end{array}\right. \\
\epsilon_{2}=\left\{\begin{array}{l}
s_{4}^{\left(j_{2}\right)} \beta_{2}^{(4)}-s_{3}^{\left(j_{2}\right)} \gamma_{2}+s_{2}-1, \\
\gamma_{2}-\beta_{2}^{(4)}-2-\delta, \\
\gamma_{2}-1-\delta,
\end{array}\right. \\
\epsilon_{3}=\left\{\begin{array}{l}
s_{3}^{\left(j_{3}\right)}-s_{4}^{\left(j_{3}\right)} \gamma_{3}-1, \\
\gamma_{3}-1-\delta,
\end{array}\right. \\
\epsilon_{4}=h-\sum_{i=1}^{3} \epsilon_{i}+g,
\end{gathered}
$$

where $g$ is the gain of the possible transfer of $n$ with the largest gain. For at most one value of $l \in\{2,3\}$ we have $\epsilon_{l}=\gamma_{l}-1$. Here $\delta=0$ or $\delta=1, j_{1}, j_{2}, j_{3} \in\{1,2, \ldots, \eta\}$.

Proof. From the $h$-range algorithm we have that the values of $\epsilon_{l}$ are given by either the conditions for the transfers to be possible or the conditions for $n$ to be in regular representation, [12]. In the algorithm we may have $p=r_{1}$, giving $L_{r_{1}+1}=\gamma_{1}$ and, from (23), $\epsilon_{1}=\gamma_{1}-2$. If $p<r_{1}$ we find $\epsilon_{1}$ from the algorithm or Theorem 1 . The possible transfer of $n$ with the largest gain and with the conditions on the $e_{j}$ such that we can have $e_{j} \leq \epsilon_{j}, j=1,2,3$, gives the gain $g \geq 0$. If no possible transfer for $n$ exists, then $g=0$.

Conjecture 1. For $k \geq 3$, there exist an $h_{s} \in \mathbf{N}$, a set of transfers $T^{(j)}=$ $\left(s_{2}^{(j)}, \ldots, s_{k}^{(j)}\right), j=1, \ldots, \eta$, and $a \sigma \in[1, \eta]$ such that for $h>h_{s}$ we have the extremal parameter basis $A_{k}^{*}(h)$ given in normal form (6) uses the transfers $T^{(j)}, j=1, \ldots, \eta$. If the regular representation of the $h$-range of the basis is

$$
n=\epsilon_{1}+\epsilon_{2} a_{2}+\ldots+\epsilon_{k} a_{k},
$$

then

$$
\begin{gathered}
\epsilon_{1}=\sum_{i=3}^{k} s_{i}^{(\sigma)} \beta_{1}^{(i)}-s_{2}^{(\sigma)} \gamma_{1}-2, \\
\epsilon_{l}=\gamma_{l}-2,
\end{gathered}
$$

for $l=2,3, \ldots, k-1$, and

$$
\epsilon_{k}=h-\sum_{i=1}^{k-1} \epsilon_{i}+g
$$

where $g$ is the gain of the possible transfer of $n$ with the largest gain.

Also from a numerical point of view the conjecture is quite interesting, to find a upper bound for a given basis.

For $k=3, h>22$, the $(0,1)$-transfer with the condition $e_{1} \geq \beta_{1}^{(3)}=\beta$, see (7), is the only transfer used for the $A_{3}^{*}$ basis, Hofmeister [4]. But with $\epsilon_{1}=\beta-2$, we cannot apply it on $n(h, 3)$. Hence, the extremal $h$-range $n(h, 3)$ is a minimal regular $h$-representation. The extremal bases for $k=3$ and $h \geq 6$ have $\epsilon_{2}=\gamma_{2}-2$. 
All the known extremal bases for $k=4$ are determined numerically and have for $h \geq 43$ and 23 other values, $6 \leq h<42, \epsilon_{2}=\gamma_{2}-2$ and $\epsilon_{3}=\gamma_{3}-2$. See Challis [2], Mossige [10] and [11].

\section{The COnjecture IN The CASE $k=4$}

Let $k=4, h=12 \alpha t+i, i \in[0,11], \alpha \geq 1, \alpha \in \mathbf{Q}, t \in \mathbf{N}$. The parameter basis $A_{M}=A_{M}(h, b, p)$ we are going to use in normal form is $\left(a_{1}=1\right)$

$$
\begin{aligned}
& a_{2}=9 \alpha t+b_{1} t+p_{1}, \\
& a_{3}=\left(3 \alpha t+b_{3} t+p_{3}\right) a_{2}-\left(5 \alpha t+b_{2} t+p_{2}\right), \\
& a_{4}=\left(2 \alpha t+b_{6} t+p_{6}\right) a_{3}-\left(\alpha t+b_{5} t+p_{5}\right) a_{2}-\left(6 \alpha t+b_{4} t+p_{4}\right),
\end{aligned}
$$

where $b_{l}, p_{l} \in \mathbf{Z}$ (to be chosen suitablely) and where we put $b=\left(b_{1}, b_{2}, \ldots, b_{6}\right)$ and $p=\left(p_{1}, p_{2}, \ldots, p_{6}\right)$. We shall also consider the basis $A_{S}=A_{S}(h, b, p)$, given by replacing the coefficient 5 in (26) by 7 and the coefficient 6 by 4 . Let $A_{M}=A_{M}(h, b)$ be the basis (26) with $p=(0, \ldots, 0)$, and similarly, $A_{S}=A_{S}(h, b)$.

Since 1971, the "record" prefactor $\varphi=2$ was held by the parameter basis $A_{M}(h, b)$ discovered by Hofmeister and Schell [5] with

$$
b=(0,0, \ldots, 0), \quad \alpha=1
$$

and the transfers that give a positive gain

$$
T_{1}=(0,1,0), \quad T_{2}=(0,0,1), \quad T_{3}=(1,1,2), \quad T_{4}=(1,0,2) .
$$

In 1988 Braunschädel [1] gave the basis $A_{S}$ with (27), using the transfers

$$
T_{1}, T_{2}, T_{3}, T_{4}^{\prime}=(0,0,2) .
$$

He examined (on a computer) all bases $A_{4}(h)$ of the form,

$$
h=H t ; a_{2}=c_{1} t, a_{3}=c_{2} t a_{2}-c_{3} t, a_{4}=c_{4} t a_{3}-c_{5} t a_{2}-c_{6} t,
$$

with $c_{l} \in \mathbf{N}$, allowing only $\left(s_{2}, s_{3}, s_{4}\right)$-transfers with $s_{2}, s_{3}, s_{4} \leq 2$. He then always found $\varphi \leq 2$, and $\varphi=2$ only for the bases $A_{M}(h, b)$ and $A_{S}(h, b)$ with (27) (see also Selmer [19]).

The author's idea was to make small variations of the leading coefficients of the elements of the basis $(26)$, by varying $b$ around the six-tuple $(0, \ldots, 0)$, to see whether an increase of the prefactor is possible. Let

$$
b_{M}=(15,1,-15,6,-13,-20) .
$$

In 1985 he found a basis $A_{M}\left(h, b_{M}\right)$ with $\varphi>2$ (see [12]):

To get the prefactor $\varphi$ of this basis we consider the polynomial

$$
g(\gamma)=-32 \gamma-168 \gamma^{2}-22 \gamma^{3}+3 \gamma^{4}
$$

and determine the solution $\gamma_{1}$ of $g^{\prime}\left(\gamma_{1}\right)=0$, where

$$
\begin{gathered}
\gamma_{1}=\frac{11}{6}+\frac{1}{3} \sqrt{457} \cos \frac{\xi+4 \pi}{3}, \cos \xi=\frac{7163}{\sqrt{457^{3}}}, \text { with } 0<\xi<\pi / 2, \\
\text { giving } \gamma_{1}=-0.09712372 \ldots
\end{gathered}
$$

With this $\gamma_{1}$ we put $\alpha_{1}=-20 / \gamma_{1}$ and

$$
\sigma=2+3^{-1} 2^{-6} g\left(\gamma_{1}\right)=2.0080397 \ldots
$$


For given $\varepsilon>0$ we can choose $t$ so large that for $h=12\left\lfloor\alpha_{1} t\right\rfloor$ the basis $A_{M}\left(h, b_{M}\right)$ has the prefactor

$$
\varphi>\sigma-\varepsilon .
$$

In fact, here $\sigma$ is a cubic irrationality, and can only be approximated by "rational" bases (26). We obtain a very good approximation if we put $\alpha=206$, that is, $h=2472 t$, giving $\sigma$ of (32) with all seven decimals correct. As usual, $\lfloor x\rfloor$ denotes the largest integer $\leq x \in \mathbf{R}$.

In [11] we developed formulas for the possible $h$-ranges of the parameter bases $A_{M}(h, b, p)$, and based the optimization on the determination of the local $h$-range $n\left(h, A_{M}\right)$. In addition to the transfers $(28)$, we discovered that it was possible to use

$$
T_{5}=(1,2,1), \quad T_{6}=(1,0,3) .
$$

In spite of the very small improvement on $\varphi=2$, this result gave quite a new situation. Let

$$
b_{S}=(15,-1,-15,2,-13,-20) .
$$

In 1988 Selmer [19] showed that also the basis $A_{S}\left(h, b_{S}\right)$ has the prefactor $(32)$. In [13] we show that my cited result $(31),(32)$ for the basis $A_{M}\left(h, b_{M}\right)$ is valid also for the basis $A_{S}\left(h, b_{S}\right)$ with $b_{M}$ replaced by $b_{S}$. Selmer [18] calls the two bases an associate pair of bases.

Computational results. When we apply our $h$-range algorithm to the parameter bases $A_{M},(26)$ and $A_{S}$, it gives for each basis the sufficient inequalities that express the conditions that all the integers $n \in[1, n(h, A)]$ have an $h$-representation and it gives all the $h$-range formula candidates. By extensive computations for $h \leq 620000$ we came to two constructions of two bases. For details see [13].

Construction 1. Given $h=12 \alpha t+i \geq 1236$, where $\alpha \in \mathbf{Q}, t \in \mathbf{N}$ and $0 \leq i \leq 11$. Let $b_{M}=(15,1,-15,6,-13,-20)$. Let $P_{i}=\left(p_{1}, p_{2}, \ldots, p_{6}\right)$ and $r$ be given by Table 1. Let $\beta=\alpha t=\lfloor h / 12\rfloor, i=h-\lfloor h / 12\rfloor 12$ and $q=r+i$. The basis $A(t)$ has the elements $\left(a_{1}=1\right)$

$$
\begin{aligned}
& a_{2}=9 \beta+15 t+p_{1}, \\
& a_{3}=\left(3 \beta-15 t+p_{3}\right) a_{2}-\left(5 \beta+t+p_{2}\right), \\
& a_{4}=\left(2 \beta-20 t+p_{6}\right) a_{3}-\left(\beta-13 t+p_{5}\right) a_{2},-\left(6 \beta+6 t+p_{4}\right),
\end{aligned}
$$

and $h$-range formula

$$
\begin{aligned}
n(t)= & (3 \beta+45 t+q+1) a_{4}+\left(2 \beta-20 t+p_{6}-2\right) a_{3} \\
& +\left(3 \beta-15 t+p_{3}-2\right) a_{2}+5 \beta+t+p_{2}-2 .
\end{aligned}
$$

Let $j=\left\lfloor\beta / \alpha_{1}\right\rfloor$, where $\alpha_{1}$ is given in the cited result (31), (32). If $i=0$ then put $j=\left\lfloor\beta / \alpha_{1}\right\rfloor+1$. If $n(j+1)>n(j)$ then $t=j+1$, else $t=j$.

Then the basis $A_{M}^{*}=A_{M}\left(h, b_{M}, P_{i}\right)=A(t)$ has $h$-range

$$
n\left(h, A_{M}^{*}\right)=n(t) .
$$

For $i$ even, $h-h_{0}=1$. For $i$ odd, $h-h_{0}=0$.

Construction 2. Given $h=12 \alpha t+i \geq 1236$, where $\alpha \in \mathbf{Q}, t \in \mathbf{N}$ and $0 \leq i \leq 11$. Let $b_{S}=(15,-1,-15,2,-13,-20)$. Let $P_{i}=\left(p_{1}, p_{2}, \ldots, p_{6}\right)$ and $r$ be given by 
TABLE 1

\begin{tabular}{r|rrrrrr|r}
$i$ & $p_{1}$ & $p_{2}$ & $p_{3}$ & $p_{4}$ & $p_{5}$ & $p_{6}$ & $r$ \\
\hline 0 & -8 & 0 & 10 & -3 & 7 & 13 & -25 \\
1,2 & -4 & 1 & 8 & -1 & 5 & 10 & -19 \\
3,4 & 0 & 2 & 6 & 1 & 3 & 7 & -13 \\
5,6 & 4 & 3 & 4 & 3 & 1 & 4 & -7 \\
7,8 & 8 & 4 & 2 & 5 & -1 & 1 & -1 \\
9,10 & 12 & 5 & 0 & 7 & -3 & -2 & 5 \\
11 & 16 & 6 & -2 & 9 & -5 & -5 & 11
\end{tabular}

TABLE 2

\begin{tabular}{r|rrrrrr|r}
$i$ & $p_{1}$ & $p_{2}$ & $p_{3}$ & $p_{4}$ & $p_{5}$ & $p_{6}$ & $r$ \\
\hline 0 & -12 & 1 & 14 & -1 & 11 & 19 & -38 \\
1,2 & -8 & 2 & 12 & 0 & 9 & 16 & -32 \\
3,4 & -4 & 3 & 10 & 1 & 7 & 13 & -26 \\
5,6 & 0 & 4 & 8 & 2 & 5 & 10 & -20 \\
7,8 & 4 & 5 & 6 & 3 & 3 & 7 & -14 \\
9,10 & 8 & 6 & 4 & 4 & 1 & 4 & -8 \\
11 & 12 & 7 & 2 & 5 & -1 & 1 & -2
\end{tabular}

Table 2. Let $\beta=\alpha t=\lfloor h / 12\rfloor, i=h-\lfloor h / 12\rfloor 12$ and $q=r+i$. The basis $A(t)$ has the elements $\left(a_{1}=1\right)$

$$
\begin{aligned}
& a_{2}=9 \beta+15 t+p_{1}, \\
& a_{3}=\left(3 \beta-15 t+p_{3}\right) a_{2}-\left(7 \beta-t+p_{2}\right), \\
& a_{4}=\left(2 \beta-20 t+p_{6}\right) a_{3}-\left(\beta-13 t+p_{5}\right) a_{2},-\left(4 \beta+2 t+p_{4}\right),
\end{aligned}
$$

and $h$-range formula

$$
\begin{aligned}
n(t)= & (3 \beta+45 t+q+1) a_{4}+\left(2 \beta-20 t+p_{6}-2\right) a_{3} \\
& +\left(3 \beta-15 t+p_{3}-2\right) a_{2}+4 \beta+2 t+p_{4}-2 .
\end{aligned}
$$

Let $j=\left\lfloor\beta / \alpha_{1}\right\rfloor$, where $\alpha_{1}$ is given in the cited result (31), (32). If $i \leq 4$, then put $j=\left\lfloor\beta / \alpha_{1}\right\rfloor+1$. If $n(j+1)>n(j)$, then $t=j+1$, else $t=j$.

Then the basis $A_{S}^{*}=A_{S}\left(h, b_{S}, P_{i}\right)=A(t)$ has $h$-range

$$
n\left(h, A_{S}^{*}\right)=n(t) .
$$

For $i$ even, $h-h_{0}=1$. For $i$ odd, $h-h_{0}=0$.

Two parameter bases $A^{(1)}(h)$ and $A^{(2)}(h)$ is said to be asymptotically equal if $a_{j}^{(1)} / a_{j}^{(2)} \rightarrow 1$ when $h \rightarrow \infty$ for $2 \leq j \leq 4$.

In an unpublished work from 1991, Kirfel and the author have shown the following result. For $h \rightarrow \infty$, all the bases $A=A(h)$ with prefactor $\varphi>2.008$ are either asymptotically equal to $A_{M}\left(h, b_{M}\right)$ or equal to $A_{S}\left(h, b_{S}\right)$. The sets $b_{M}$ and $b_{S}$ are given in (31) and (34) respectively. In [12] it is shown that such bases exist.

Let $\mathcal{A}_{M}\left(h, b_{S}\right)$ denote the class of all bases $A=A(h)$ that are asymptotically equal to $A_{M}\left(h, b_{M}\right)$, and let $\mathcal{A}_{S}\left(h, b_{S}\right)$ be the similar class for $A_{S}\left(h, b_{S}\right)$.

We now ask for the best choice of the basis $A=A(h)$ in the class $\mathcal{A}_{M}\left(h, b_{M}\right)$ and the best choice of the basis in $\mathcal{A}_{S}\left(h, b_{S}\right)$. 
Extensive computations for $h \leq 620000$ give the following results.

Result $A$. For $9793 \leq h \leq 620000, A_{M}^{*}=A_{M}(h)$ in the class $\mathcal{A}_{M}\left(h, b_{M}\right)$ is the basis with the largest $h$-range.

Result B. For $10653 \leq h \leq 620000, A_{S}^{*}=A_{S}(h)$ in the class $\mathcal{A}_{S}\left(h, b_{S}\right)$ is the basis with the largest $h$-range.

Result C. For $11385 \leq h \leq 620000, A_{M}^{*}$ has larger $h$-range than $A_{S}^{*}$.

For both the bases $A_{M}^{*}$ and $A_{S}^{*}$ the $h$-range formulas are of the type stated in Conjecture 1.

Conjecture 2. For $h \geq 11385, A_{M}^{*}=A_{M}(h)$ is the extremal basis.

For $h<11385$ we may get better bases when we replace the set $b_{M}$ or $b_{S}$ by other sets $b$ and suitable sets $p$.

Using a result of Selmer [18], we prove in [13] that if $b=\left(b_{1}, \ldots, b_{6}\right)$ and

$$
b^{\prime}=\left(b_{1}, b_{1}-b_{2}+b_{3}, b_{3}, b_{1}-b_{4}-b_{5}+b_{6}, b_{5}, b_{6}\right),
$$

then for each basis $A_{M}(h, b)$ with prefactor $\varphi$ there is a basis $A_{S}\left(h, b^{\prime}\right)$ with the same prefactor, and vice versa. If $b=b^{\prime}$, then

$$
2 b_{2}=b_{1}+b_{3}, \quad 2 b_{4}=b_{1}-b_{5}+b_{6} .
$$

For further details see [13].

\section{REFERENCES}

[1] R. Braunschädel, Zum Reichweitenproblem, Diplomarbeit, Math. Inst., Joh. GutenbergUniv., Mainz 1988

[2] M.F.Challis, Two new techniques for computing extremal $h$-bases $A_{k}$, Computer J. 36 (1993), 117-126.

[3] G. Hofmeister, Über eine Menge von Abschnittbasen, J. Reine Angew. Math. 213 (1963) 43-57. MR 31:149

[4] G. Hofmeister, Asymptotische Abschätzungen für dreielementige Extremalbasen in natürlichen Zahlen, J. Reine Angew. Math. 232 (1968) 77-101. MR 38:1068

[5] G. Hofmeister, Zum Reichweitenproblem, Mainzer Seminarberichte in Additiven Zahlentheorie, 1 (1983), 30-52.

[6] G. Hofmeister, C. Kirfel and H. Kolsdorf, Extremale Reichweiten, Inst. Rep. No 60, Dept. of pure Math., Univ. of Bergen, 1991.

[7] C. Kirfel, On Extremal Bases for the $h$-range Problem, I, II, Inst. Rep. Nos. 53, 55, Dept. of pure Math., Univ. of Bergen, 1989, 1990

[8] C. Kirfel, Extremale asymptotische Reichweitenbasen, Acta Arith. (1992) 279-288. MR 92m:11012

[9] W.F. Lunnon, A Postage Stamp Problem, Compt. J. 12 (1969), 377-380. MR 40:6745

[10] S. Mossige, Algorithms for computing the $h$-range of the Postage Stamp Problem, Math. Comp., 36 (1981), 575-582. MR 82e:11095

[11] S. Mossige, On the extremal $h$-range of the Postage Stamp Problem with four Stamp denominations, Dissertation, Inst. Rep. No. 41, Dept. of pure Math., Univ. of Bergen, 1986.

[12] S. Mossige, On extremal $h$-bases $A_{4}$, Math. Scand. 61 (1987), 5-16. MR 89e:11008

[13] S. Mossige, The Postage Stamp Problem. An algorithm to determine the $h$-range. On the $h$-range formula. On the extremal basis problem for $k=4$, Inst. Rep. No. xx, Dept. of pure Math., Univ. of Bergen, 1995, 1-74.

[14] A. Mrose, Die Bestimmung der extremalen regulären Abschnittbasen mit Hilfe einer Klasse von Kettenbruchdeterminanten, Dissertation, Freie Universität Berlin, 1969.

[15] $\varnothing$. Rødseth, An upper bound for the $h$-range of the Postage Stamp Problem, Acta Arith. 54 (1990), 301-306. MR 91h:11013 
[16] J. Riddell and C. Chan, Some extremal 2-bases, Math. Comp. 32(1978), 630-634. MR 57:16244

[17] E.S. Selmer, The Local Postage Stamp Problem, I-III, Inst. Rep. Nos. 42, 44, 47, Dept. of Pure Math., Univ. of Bergen, 1986, 1990.

[18] E.S. Selmer, Associate Bases in the Postage Stamp Problem, J. Number Theory, 42, 3,(1992), 320-336. MR 94b:11012

[19] E.S. Selmer, Asymptotic $h$-ranges and dual bases, Inst. Rep. No. 56, Dept. of Pure Math., Univ. of Bergen, 1990.

[20] A. Stöhr, Gelöste und ungelöste Fragen über Basen der natürlichen Zahlenreihe, I, J. Reine Angew. Math. 194 (1955), 40-65. MR 17:713a

University of Bergen, Department of Mathematics, Joh. Brunsgt. 12, N-5008 Bergen, NORWAY

E-mail address: svein.mossige@mi.uib.no 